\title{
Baseline assessment of antibiotics prescribing for acute, non- complicated infections in primary care in Germany in the cluster randomized trial ARena
}

\author{
Regina Poss-Doering ( $\square$ regina.poss-doering@med.uni-heidelberg.de ) \\ University Hospital Heidelberg https://orcid.org/0000-0003-0618-4034 \\ Dorothea Weber \\ University Hospital Heidelberg, Institute for Medical Biometry \\ Martina Kamradt \\ University Hospital Heidelberg, Dept. of General Practice and Health Services Research \\ Edith Andres \\ aQua Institut, Goettingen \\ Petra Kaufmann-Kolle \\ aQua Institut, Goettingen \\ Joachim Szecsenyi \\ The University of Melbourne Department of General Practice
}

\section{Research}

Keywords: antimicrobial resistance, antibiotic prescribing, acute non-complicated infections, primary care, mixed logistic regression model

Posted Date: October 30th, 2020

DOI: https://doi.org/10.21203/rs.3.rs-59028/v2

License: (c) (i) This work is licensed under a Creative Commons Attribution 4.0 International License. Read Full License 


\section{Abstract}

Background Antimicrobial resistance is fueled by inappropriate prescribing and use of antibiotics. Global and national strategies support rational and adequate use of antibiotics to retain treatment options and fight resistances. In Germany, the ARena project (Sustainable reduction of antibiotic-induced antimicrobial resistance) was intended to promote the rational and appropriate use of antibiotics for acute non-complicated infections by addressing physicians, care teams and patients through multiple interacting interventions. This paper presents patterns of antibiotics prescribing for patients with acute non-complicated infections in participating primary care networks prior to the start of the ARena project, explores variation across subgroups of patients and draws comparisons to reference groups which represent standard care.

Methods In mixed logistic regression models, we explored factors associated with the primary outcome defined as the proportion of patients with acute non-complicated infections consulting primary care practices who received an antibiotic prescription. Secondary outcomes concerned the prescription of different types of antibiotics. Descriptive methods were used to summarize the data referring to primary care networks, reference groups, and subgroups.

Results Across all observed cases, antibiotic prescription rates were $31.7 \%$ in reference groups and $32.0 \%$ in primary care networks. Being the largest group of physicians observed, General practitioners prescribed antibiotics more frequently than other medical specialist groups (otolaryngologists vs. General practitioners $\mathrm{OR}=0.465 \mathrm{Cl}=[0.302 ; 0.719], \mathrm{p}<0.001$, pediatricians vs. General practitioners: $\mathrm{OR}=0.369 \mathrm{Cl}=$ $[0.135 ; 1.011], p=0.053)$. Quinolone prescription rates were moderate ( $8.1 \%$ in reference groups and $9.9 \%$ in primary care networks). Patients with comorbidities had a higher likelihood of receiving an antibiotic and quinolone prescription and were less likely to receive a recommended substance. Younger patients were less likely to receive antibiotics $(\mathrm{OR}=0.771 \mathrm{Cl}=[0.636 ; 0.933], \mathrm{p}=0.008)$. Female gender was associated with higher rates of antibiotic prescriptions compared to males (OR=1.293 Cl=[1.201, 1.392], $p<0.001)$.

Conclusion Prior to the ARena project, observed antibiotic prescription rates for acute non-complicated infections were moderate, but there was still room for improvement. The use of recommended substances was low which indicates a need for creating stronger awareness of guideline-conform use of antibiotics.

\section{Background}

The use of antibiotics in German primary care is moderate, but there is still potential for lowering prescribing rates for specific conditions. In light of growing antimicrobial resistances, the national strategy DART 2020 follows the One Health approach in Germany to counteract microbial resistance and preserve treatment options [1]. Monitoring the use of antibiotics is an important tool that serves to reduce the spreading of antimicrobial resistance, particularly in primary care where about $85 \%$ of the used antibiotics in Germany are prescribed [2]. These prescriptions are written by General Practitioners [3] in $41 \%$ of consultations for acute respiratory tract infections (ARTI), which is in accordance with prevailing clinical recommendations in $52 \%$ of these consultations [4].

In recent years, several research projects tested strategies that aim to enhance the appropriate use of antibiotics in healthcare. In this context, the ARena (Sustainable reduction of antibiotic-induced antimicrobial resistance) project (conducted from 2017 to 2020) aimed to foster the rational and appropriate use of antibiotics for acute non-complicated infections in primary care in Germany [5]. By applying a multifaceted strategy with multiple interacting intervention components, ARena addressed primary care physicians, care teams as well as patients [5].

An innovative aspect of ARena was its embedding in 14 primary care networks (PCNs) across two German federal states. These networks support primary care practices with respect to practice management and quality improvement, thus they were expected to amplify the impact of the ARena implementation program. The program was evaluated in a three-armed, cluster randomized trial. All intervention components were based on published research and experiences in quality improvement programs. To achieve its' goals, ARena addressed aspects of effective communication, education and training with physicians and care teams in the participating PCNs as well as the regional public. More detailed description of the study design and interventions can be found in the published study protocol [5].

Previous research investigated the development of annual antibiotic prescription density in primary care and found a relatively stable utilization in Germany from 2008 to 2014 [6]. However, strong variations of overall and age-group-specific distributions of antibiotic subgroups could be identified [7]. A strong awareness of antimicrobial resistance has been observed among German General Practitioners (GPs), while measures to improve rational prescribing were found to be not widely implemented [8]. In more recent research, it was found that the use of antibiotics in German primary care showed large variations between and within medical specialties and season and that a considerable proportion of antibiotic prescriptions lacked conformity with national guideline recommendations [9]. Though cited study 
findings might not reflect the most current situation or guideline-conformity of prescriptions, there is an indication that antibiotics are still not prescribed appropriately regarding indication and spectrum and thus are used inappropriately to a larger extent. For targeted quality improvement, it is relevant to know which subgroups of patients and physicians are at highest risk of inappropriate utilization of antibiotics.

For the ARena study, the outcome evaluation is based on claims-data and references established indicators of the European Surveillance of Antimicrobial Consumption Network (ESAC-Net) [10] which were tailored to the specifics of this study. Primary and secondary outcomes are related to general prescribing of systemic and local antibiotics as well as indication-specific prescribing of currently guidelinerecommended antimicrobials $[11,12]$. To explore the actual antibiotics prescribing rates and their determinants, this present study aimed to present patterns of antibiotics prescribing for patients with acute non-complicated infections prior to the start of the ARena project, explore the variation across subgroups of patients and compare this baseline to reference groups (RGs) which represent standard care.

\section{Methods}

Study design

The underlying study (Trial registration: ISRCTN, ISRCTN58150046) is a prospective observational trial with fourteen PCNs and RGs that reflect standard care in two German federal states (Bavaria and North Rhine-Westphalia). The ARena-study was approved by the ethics committee of the Medical Faculty of the University of Heidelberg (reference number: S-353/2017). The study was planned with an intervention period of 24 months, and with two parts of evaluation: (a) an outcome evaluation based on claims-data and (b) a process evaluation based on surveys.

Study population

Fourteen PCNs situated in two German federal states (Bavaria and North Rhine-Westphalia) were recruited to participate in the ARena study. For administrative reasons, the focus was on their patients insured by AOK health insurance and registered within a specific healthcare delivery program (defined by German law § 140a SGB V a.F. and § 140a Abs. 1. S. 2 Alt. 1 SGB V n.F). At baseline, approximately 40000 patients with AOK health insurance were registered in 196 participating primary care practices in these 14 networks. Medical specialties included were general practice, otolaryngologist, pediatricians, urology and gynecology. The patient population for the three intervention arms comprised patients who sought primary care for one of the following reasons: acute non-complicated infections (upper respiratory tract infections (URTI), bronchitis, sinusitis, tonsillitis and otitis media). Thus, study populations differed per indexed consultation reason regarding number of cases, age of patients and insurance status. The diagnoses were based on physician-recorded ICD codes in administrative data provided for quarterly reimbursement periods. Each physician-recorded ICD code for the defined index diagnoses represents a case, where each patient can produce multiple cases. A patients' case is recorded for each ICD code within each quarter. Written informed patient consent was prerequisite for participation in the study in North Rhine-Westphalia.

\section{Measures}

The primary outcome is the proportion of patients who were treated with systemic or local antibiotics of all patients with acute noncomplicated infections consulting primary care practices. More precise, patients suffering from acute bronchitis (18-75 years), sinusitis (> 18 years), otitis media ( $>2$ years), acute URTI ( $>1$ year), or tonsillitis ( $>1$ year) are considered in the primary outcome. Specifically excluded are diagnoses for streptococcal tonsilitis and other pathogen-caused acute forms of tonsillitis that warrant antibiotic therapy. (Additional file 1, Supplementary Table 1 details included diagnoses and related ICD codes, Supplementary Table 2 provides a list of the excluded diagnoses.)

The following secondary outcomes were examined:

1. Proportion of patients with acute non-complicated infections receiving a quinolone prescription when consulting primary care practices (patients suffering from acute bronchitis (18-75 years), sinusitis ( $>18$ years), otitis media ( $>2$ years), URTI ( $>1$ year), or tonsillitis (>1 year)).

2. Proportion of patients with acute URTI (>1 year) receiving a prescription for recommended antibiotics (amoxicillin).

3. Proportion of patients with acute bronchitis (18-75 years) receiving a prescription for recommended antibiotics (amoxicillin, tetracycline, macrolides).

4. Proportion of patients with sinusitis (> 18 years) receiving a prescription for recommended antibiotics (amoxicillin, cefuroxime).

5. Proportion of patients with tonsillitis (>1 year) receiving a prescription for recommended antibiotics (penicillin, erythromycin). 
6. Proportion of patients with otitis media (>2 years) receiving a prescription for recommended antibiotics (amoxicillin, erythromycin, cefuroxime).

7. Consumption of broad-spectrum antibiotics in DDD\% (on practice level)

(penicillin and beta-lactamase inhibitors, cephalosporines of 2nd, 3rd and 4th generation, macrolides (excluding Erythromycin) and gyrase inhibitors (fluoroquinolones).

Categorization of recommended antibiotics is based on existing evidence-based clinical guidelines developed by the German College of General Practitioners and Family Physicians (DEGAM) [12] and the Association of the Scientific Medical Societies in Germany (AWMF) [11]. (See Additional file 1, Supplementary Table 3 for currently recommended and alternative antibiotics.)

Regarding patients, the following sociodemographic, disease, and treatment characteristics in the claims data provided by AOK were included: age, sex, Charlson comorbidity index (CCI) (predicts 1-year survival in patients based on sum of relevant comorbidities [13, 14]), employment status, nationality (missing values aggregated to 'other'), insurance status (main member, family, retiree), participation in a disease management program (DMP) (type 1 diabetes, type 2 diabetes, coronary heart disease, breast cancer, bronchial asthma, COPD, cardiac insufficiency), classified degree of necessary nursing care [15], and season. Regarding primary care practices, type of location (urban, urbanized, countryside), type of practice (single or group) and medical specialty group are documented.

\section{Data analysis}

The baseline data of practices participating in ARena were analyzed regarding the four quarters prior to the ARena intervention (Q3 2016 Q2 2017) with a focus on prescription rates of antibiotics for acute non-complicated self-limiting infections and the comparison between characteristics of PCNs including their member physicians and RGs that reflect usual care. In addition, patients' sociodemographic and disease-specific characteristics in either of these groups are summarized. Infections considered are tonsillitis, sinusitis, otitis media, bronchitis, and URTI. Also focused are the use of guideline-recommended indication-appropriate antibiotics and the group of quinolones.

The primary and all secondary outcomes, as well as all documented data (patient characteristics, disease characteristics, treatment data, and practice characteristics), were first analyzed descriptively. For continuous variables, mean and standard deviation, median, 25\%/75\%quantiles [Q1-Q3], min and max are provided, for categorical variables absolute and relative frequencies are given. Note that the description of patients and disease characteristics, as well as treatment data and practice characteristics differ between endpoints, because the considered patients are defined for each endpoint by the respective disease and prescription of antibiotics. Therefore, the descriptive analysis is done for each outcome individually. For the subgroups gender, DMP and the $\mathrm{CCl}$, the primary and all secondary outcomes are contrasted using descriptive methods based on patients in PCNs and RGs.

A logistic mixed effects regression model was used to identify factors influencing the primary outcome. The model considers the nested structure of the data with patients nested in practices, which means practice is included as random effect in the logistic mixed effects model. As fixed effects, medical specialty group (Fachgruppe - FGR), urbanization, age group, sex, and the $\mathrm{CCl}$ as indication of health status are considered. Secondary outcomes are analyzed using mixed logistic (1.-6.) or beta regression models (7.). Adjustment is done as described for the primary outcome model. Since this is an explorative study, all p-values do not have confirmatory value.

\section{Results}

The primary analysis considered a total of 3129289 cases in PCNs ( $n=18$ 207) and RGs ( $n=3111$ 082) diagnosed with one of the acute non-complicated infections (tonsillitis, sinusitis, otitis media, bronchitis, and URTI). In PCNs, $92.7 \%$ of the participating practices were General Practitioners, $4.9 \%$ Otolaryngologists, and $2.1 \%$ were Pediatricians. In RGs, $73.3 \%$ were General Practitioners, $10.6 \%$ Otolaryngologists and 14.5\% were Pediatricians. The total number of observed cases mentioned above (n=3 129 289) reflects 2102783 patients with a mean of 1.5 cases per patient. In PCNs, $62.8 \%$ of the included cases were seen in rural area practices compared to $32.6 \%$ of cases in the RGs. Mean age of patients was higher in PCNs. In both groups, sex was equally distributed. Patient nationality was not reported for less than $1 \%$ of the cases and all cases without this information are considered in the category "other". A main group difference in terms of insurance status was apparent in the subgroup of retired insurance members (RGs: $10.3 \%$; PCNs $20.8 \%$ ). Sociodemographic characteristics of patients in PCNs and RGs are presented in Table 1.

\section{Discussion}


This study explored actual prescribing rates and aimed to assess and present patterns of antibiotic prescribing for patients with acute noncomplicated infections prior to the start of the ARena project. In about a third of all observed cases with acute non-complicated infections, patients received an antibiotic prescription. It is known that rates of antibiotic prescriptions in German primary care overall have been decreasing constantly between 2010 and 2018, particularly with regards to children and adolescents [20] who were underrepresented in this study sample. Room for improvement can be expected to be more visible in 'high prescribers', but since the potential for improvement noticeably decreased in recent years already, observed effects of the ARena project might appear to be small when assessed in the pending final analysis of intervention effectiveness.

A recent national cross-sectional study in USA identified that $57 \%$ of 130.5 million prescriptions for antibiotics written during ambulatory care visits in 2015 were for appropriate indications, $25 \%$ were inappropriate and $18 \%$ had no documented indication. It was noted that being an adult male, spending more time with the provider and seeing a non-primary care specialist were significantly positively associated with non-indicated antibiotic prescriptions [21]. In contrast to these findings, our data indicate that antibiotics were most frequently prescribed by general practitioners. In addition, the prescription rate of broad-spectrum antibiotics showed slightly higher rates in PCNs compared to RGs. These discrepancies in antibiotic prescription rates between PCNs and RGs might be explained by the differing specialist group distribution between PCNs and RGs and a small percentage of specialists and a higher percentage of GPs in PCNs than RGs. A contributing factor towards the slight difference in prescription rates between PCNs and RGs can also be seen in the higher patient age and morbidity in PCNs which is in line with the findings of Shaver et al. [22]. The exception here were prescriptions for Otitis media where cases were included from the age of two and above and therefore morbidity considerations cannot deliver explanations for higher prescription rates in PCNs.

The prescription rates for quinolones were generally moderate to low in PCNs (9.9\%) and RGs (8.1\%) and thus match recent findings of a study that examined antibiotic prescribing in the US outpatient setting where $9.4 \%$ of investigated visits for acute respiratory infections received a broad-spectrum antibiotic [22]. Prescribing quinolones seemed to be associated with a higher number of comorbidities and higher patient age as well which complements findings of the process evaluation conducted alongside ARena where uncertainty about diagnoses, prognosis, continuity of care and perceptions about patient preferences were found to be among the reasons for non-indicated prescriptions [23]. To some extend this confirms findings from a 2010 study that investigated fluoroquinolone prescriptions for acute cough in German primary care to find predictors for unjustified prescriptions. The researchers then concluded that unjustified quinolone prescriptions were determined by patient characteristics such as severity and duration of illness and patient age. However, they also found that physicians with higher individual antibiotics prescribing rates and physicians with hospital-based specialty training - versus combined hospital and ambulatory training - were more likely to prescribe fluoroquinolones than physicians who specifically trained as GPs [24]. Interestingly, our data showed that in urbanized PCN practice locations, quinolone prescription rates were lower than in rural locations. This complements results from a previous study where German GPs working in urban areas were found to be more likely to use the strategy of delayed prescribing of antibiotics than GPs working in rural areas [10]. Such discrepancies between urban and rural prescribing habits potentially could root in more ample opportunities for self-reflection about prescribing motivated by regular peer exchange and a broader and more frequent offer of continued training and upskilling in urban locations. Efforts to educate physicians, care teams and patients continuously about current and appropriate diagnosis and therapy options as well as communication about them may remedy this to some extent.

The perceived, but not actually communicated patient request for antibiotics is often overrated by physicians [25] and may initiate unfounded assumptions of losing patients to another physician when therapy followed guideline recommendations and antibiotics are not prescribed. In this study, the rate of recommended antibiotics was remarkably low and in line with the high percentage of broad-spectrum antibiotics on practice level which is another potential indicator for physicians' uncertainty. Patients with higher comorbidity and patients participating in DMPs were more likely to get a quinolone prescription, and less likely to receive one of the recommended substances. This might be related to the higher age and poorer health status of these patients as supported by previous research [22], but also to the physicians' striving for the elimination of both their own and patients' insecurities and potential complications. One effective way to eliminate insecurities and reduce prescribing antibiotics was found to be the promotion of communication skills by means of a short communication training for primary care physicians. In a recent study in Germany, results showed a prescribing probability decrease of 6.5 percentage points for the treatment of URTI and an even stronger impact for female patients aged below 35 [26]. As the ARena project also used an intervention component to strengthen communication skills, similar effects can be expected.

The rate of prescribed broad-spectrum antibiotics in PCNs in this study seemed slightly higher compared to a previous study [22]. However, for this present study the outcome was assessed by DDD\% on practice level whereas Shaver et al. evaluated on patient level. Factors associated with the prescription of recommended antibiotics for the considered infections could not be clearly identified. Information about patient nationality was not available for all cases and the percentage of nationalities other than German is low. However, our data showed 
that the percentage of observed cases of Eastern European patients was higher than the one of Southern Europeans in PCNs as well as in RGs. This is contrasted by findings of the process evalaution in ARena where physicians indicated their subjective perception of many Southern Europeans asking for antibiotics prescriptions [23].

\section{Strengths and limitations}

One strength of this study is the careful exclusion of patients in case there is an ICD code documented which justifies the antibiotic prescription. Additionally, the type of antibiotics (recommended, quinolones, broad-versus small-spectrum) is analyzed in detail. Limitations of this observational study of baseline data is the restriction on ICD Codes and claims-related health insurance data. Therefore disease, patient, and practice information are limited. Direct connection between ICD Code and prescription of antibiotics is not possible via claims data, thus ICD codes and antibiotics prescription were matched by quarter which introduces a potential bias. The use of DDDs for pediatric antibiotic consumption where weight-based dosing is appropriate, is an additional limitation. Participating practices and PCNs were already recruited for the ARena trial and might be more alert to the topic and supportive of appropriate prescribing already (shared attitude in the networks that might only be attractive for certain physicians). So far only one time point before the planned intervention in the ARena trial could be analyzed. Final assessment of effects will be possible through the analysis of post intervention data with a focus on the evaluation of the interventions. Findings of this present study can then serve as comparator in the final report of the outcome evaluation.

\section{Conclusion}

Antibiotics prescription rates for acute non-complicated infections were moderate prior to the intervention start of the ARena project, but still not sufficiently appropriate. Guideline-recommended substances were underrepresented and the prescription rate of broad-spectrum antibiotics was alarming. This indicates a need for creating stronger awareness of guideline-conform use of antibiotics as intended by the implementation program used in ARena. The final outcome evaluation will use this baseline evaluation as comparator and inform about potential effects of the program.

\section{Abbreviations}

ARTI - acute respiratory tract infections

AWMF - Association of the Scientific Medical Societies in Germany

CAP - community acquired pneumonia

$\mathrm{Cl}$ - confidence interval

$\mathrm{CCl}$ - Charlson comorbidity Index

DDD - defined daily dose

DEGAM - German College of General Practitioners and Family Physicians

DMP - disease management program

ESAC-Net - European Surveillance of Antimicrobial Consumption Network

GP - general practitioners

ICD - International Classification of Diseases

OR - odds ratio

PCNs - primary care networks

Q - quarter

RG - reference group reflecting standard care 


\section{Declarations}

\section{Ethics approval and consent to participate}

This study received ethical approval by the medical ethics committee of the Medical Faculty of Heidelberg University (S-353/2017) and the ethics committee of the medical Association Baden-Wurttemberg (B-F-2017-104). Participants in this study all gave written informed consent for participation and publication of findings. Confidentiality and anonymity were ensured throughout the study. Trial registration: ISRCTN, ISRCTN58150046

\section{Availability of data and material}

All analyses generated for this study are included in this published article and its supplementary information files 1 and 2 . The original datasets that support the findings of this study are not publicly available due to restrictions stipulated by German law and the data provider. AOK can be contacted for access to the data.

\section{Competing interests}

SZ is founder and managing director of the aQua Institut, Goettingen and Medical Director at the University Hospital Heidelberg, Germany, Department of General Practice and Health Services Research. The authors declare that they have no competing interests.

\section{Funding}

The ARena study received funding from the Innovation Committee at the Federal Joint Committee (G-BA), Berlin (01NVF16008). The funder had no role in the design, data-collection, data-analysis, interpretation, or writing of the paper.

\section{Author's contributions}

RPD and DW drafted and prepared the manuscript. DW, RPD, and MW contributed to concept and desaf this study. DW, RPD, MK, MW, EA, and PKK collaborated on the construction of the statistical analysis plan. DW analyzed the baseline data. DW, RPD and MW interpreted the data. SZ was overall principal investigator of the ARena project. All authors provided substantial comments and approved the final version of the manuscript.

RPD - Regina Poss-Doering; DW - Dorothea Weber; MK - Martina Kamradt; EA - Edith Andres; PKK - Petra Kaufmann-Kolle; MW - Michel Wensing; SZ - Joachim Szecsenyi

\section{Acknowledgements}

The authors would like to thank the aQua Institute and their staff for taking the lead in the ARena consortium. In particular, we thank Linda Barnewold, aQua Institut, for supporting data management in this study and Andreas Gutscher, Gitta Friedrichs und Yvonne Giesler, aQua Institut, for their administrative support. We would also like to thank all involved ARena project partners for their advice, contributions and overall support in conducting the ARena study: Agency of German Practice Based Physicians (Agentur deutscher Arztnetze), statutory health insurers AOK Bayern, AOK Rheinland-Hamburg, and Federal AOK Association (AOK Bundesverband), and the Bavarian Association of Statutory Health Insurance Physicians (KV Bayerns).

**ARena-study group: Joachim Szecsenyi, Michel Wensing, Martina Kamradt, Regina Poß-Doering, Dept. of General Practice and Health Services Research, University Hospital Heidelberg; Dorothea Weber, IMBI Institute for Medical Biometry, Heidelberg; Petra Kaufmann-Kolle, Edith Andres, aQua Institut, Goettingen; Non-author contributors: Dr. Veit Wambach, AdA Agency for German Practice Based Physicians, Berlin; Joerg Lindenthal, QuE Nuremberg; Julian Bleek, Federal AOK Association, Berlin; Alexander Günter, statutory health insurer AOK 
Bavaria (AOK Bayern), Lutz Bader, Bavarian Association of Statutory Health Insurance Physicians (KV Bayerns) served as scientific advisors throughout the ARena study.

\section{References}

1. European Centre for Disease Prevention Control. Antimicrobial resistance surveillance in Europe 2015. Annual Report of the European Antimicrobial Resistance Surveillance Network (EARS-Net): Annual report of the European Antimicrobial Resistance Surveillance Network (EARS-Net) 2015. Stockholm: ECDC; 2017 [23.04.2019].

2. European Centre for Disease Prevention Control. Surveillance of antimicrobial consumption in Europe 2013-2014. Stockholm: European Centre for Disease Prevention and Control; 2018 [23.04.2019]; Available from: https://ecdc.europa.eu/sites/portal/files/documents/Surveillance-antimicrobial-consumption-Europe-ESAC-Net-2013-14.pdf.

3. German Federal Ministry of Health. DART 2020 | 2nd Interim Report 2017. online: German Federal Ministry of Health. Federal Ministry of Food and Agriculture. Federal Ministry of Education and Research. ; 2017; Available from: https://www.bundesgesundheitsministerium.de/fileadmin/Dateien/3_Downloads/D/DART_2020/DART2020_Interim_Report_2017.pdf.

4. Federal Office of Consumer Protection and Food Safety P-E-GfCeV. Germap 2015: Antimicrobial Resistance and Consumption; Report on the consumption of antimicrobials and the spread of antimicrobial resistance in human and veterinary medicine in Germany. Rheinbach: Antiinfectives Intelligence, 2016.

5. German Federal Ministry of Health. Bundesministerium für Gesundheit. DART 2020 - Antibiotika-Resistenzen bekämpfen zum Wohl von Mensch und Tier. Berlin: Bundesministerium für Gesundheit, 2015.

6. Kraus EM, PelzI S, Szecsenyi J, Laux G. Antibiotic prescribing for acute lower respiratory tract infections (LRTI) - guideline adherence in the German primary care setting: An analysis of routine data. PloS one. 2017;12(3):e0174584. doi: 10.1371/journal.pone.0174584.

7. Kamradt M, Kaufmann-Kolle P, Andres E, Brand T, Klingenberg A, Glassen K, et al. Sustainable reduction of antibiotic-induced antimicrobial resistance (ARena) in German ambulatory care: study protocol of a cluster randomised trial. Implementation Science. 2018 2018/02/05;13(1):23. doi: 10.1186/s13012-018-0722-0.

8. Bätzing-Feigenbaum J, Schulz M, Schulz M, Hering R, Kern WV. Outpatient Antibiotic Prescription. Dtsch Arztebl International. 2016 July 1, 2016;113(26):454-9. doi: 10.3238/arztebl.2016.0454.

9. Holstiege J, Schink T, Molokhia M, Mazzaglia G, Innocenti F, Oteri A, et al. Systemic antibiotic prescribing to paediatric outpatients in 5 European countries: a population-based cohort study. BMC Pediatr. 2014 Jul 5;14:174. PMID: 24997585. doi: 10.1186/1471-2431-14174.

10. Salm F, Schneider S, Schmücker K, Petruschke I, Kramer TS, Hanke R, et al. Antibiotic prescribing behavior among general practitioners - a questionnaire-based study in Germany. BMC infectious diseases. 2018;18(1):208. doi: 10.1186/s12879-018-3120-y.

11. Schwartz KL, Brown KA, Etches J, Langford BJ, Daneman N, Tu K, et al. Predictors and variability of antibiotic prescribing amongst family physicians. J Antimicrob Chemother. 2019 Jul 1;74(7):2098-105. PMID: 31002333. doi: 10.1093/jac/dkz112.

12. Zweigner J, Meyer E, Gastmeier P, Schwab F. Rate of antibiotic prescriptions in German outpatient care - are the guidelines followed or are they still exceeded? GMS hygiene and infection control. 2018;13:Doc04. doi: 10.3205/dgkh000310.

13. Adriaenssens N, Coenen S, Versporten A, Muller A, Minalu G, Faes C, et al. European Surveillance of Antimicrobial Consumption (ESAC): outpatient antibiotic use in Europe (1997-2009). J Antimicrob Chemother. 2011;66 Suppl 6:vi3-12. PMID: 22096064. doi: $10.1093 / \mathrm{jac} / \mathrm{dkr} 453$.

14. Association of the Scientific Medical Societies in Germany (AWMF). Aktuelle Leitlinien (Current guideliens). online: AWMF.

15. German College of General Practitioners and Family Physicians DEGAM. Leitlinien der DEGAM. [20.06.2020]; Available from: https://www.degam.de/degam-leitlinien-379.html. 
16. Charlson ME, Pompei P, Ales KL, MacKenzie CR. A new method of classifying prognostic comorbidity in longitudinal studies: development and validation. J Chronic Dis. 1987;40(5):373-83. PMID: 3558716. doi: 10.1016/0021-9681(87)90171-8.

17. Quan H, Sundararajan V, Halfon P, Fong A, Burnand B, Luthi JC, et al. Coding algorithms for defining comorbidities in ICD-9-CM and ICD-10 administrative data. Med Care. 2005 Nov;43(11):1130-9. PMID: 16224307. doi: 10.1097/01.mlr.0000182534.19832.83.

18. Zweites Gesetz zur Stärkung der pflegerischen Versorgung und zur Änderung weiterer Vorschriften (zweites Plegestärkungsgesetz (PSG II), (2015).

19. Online-Ratgeber Pflege. Pflegegrade., (2018).

20. Holstiege J SM, Akmatov MK, Steffen A, Bätzing J Outpatient use of systemic antibiotics in Germany from 2010 to 2018-A population-based study. Berlin: 2019.

21. Ray MJ, Tallman GB, Bearden DT, Elman MR, McGregor JC. Antibiotic prescribing without documented indication in ambulatory care clinics: national cross sectional study. Bmj. 2019 Dec 11;367:I6461. PMID: 31826860. doi: 10.1136/bmj.16461.

22. Shaver AL, Jacobs DM, LaMonte MJ, Noyes K. Antibiotic prescribing for acute respiratory tract infections in the United States outpatient setting. BMC Fam Pract. 2019;20(1):91-. PMID: 31266449. doi: 10.1186/s12875-019-0980-1.

23. Poss-Doering R, Kamradt M, Stuermlinger A, Glassen K, Kaufmann-Kolle P, Andres E, et al. The complex phenomenon of dysrational antibiotics prescribing decisions in German primary healthcare: a qualitative interview study using dual process theory. Antimicrobial Resistance \& Infection Control. 2020 2020/01/06;9(1):6. doi: 10.1186/s13756-019-0664-6.

24. Altiner A, Wilm S, Wegscheider K, Sielk M, Brockmann S, Fuchs A, et al. Fluoroquinolones to treat uncomplicated acute cough in primary care: predictors for unjustified prescribing of antibiotics. J Antimicrob Chemother. 2010 Jul;65(7):1521-5. PMID: 20494927. doi: $10.1093 / \mathrm{jac} / \mathrm{dkq} 151$.

25. Klingenberg A BT, Andres E, Kaufmann-Kolle P, Wambach V, Szecsenyi J. Was wissen Patienten über Antibiotika, und wie häufig erwarten sie deren Verordnung? Zeitschrift für Allgemeinmedizin ZFA. 2019;05/19(Deutscher Ärzteverlag Z Allg Med 2019; 95 (5)). doi: DOI: 10.3238/zfa.2019.0198-0202.

26. Strumann C, Steinhaeuser J, Emcke T, Sönnichsen A, Goetz K. Communication training and the prescribing pattern of antibiotic prescription in primary health care. PLoS One. 2020;15(5):e0233345. PMID: 32428012. doi: 10.1371/journal.pone.0233345.

\section{Supplementary Files}

This is a list of supplementary files associated with this preprint. Click to download.

- CONSORT2010Checklist20200812.docx

- 20200807AppendixARenaBaselineSuppl2final.docx

- 20200807AppendixARenaBaselineSuppl1final.docx 